\title{
Microwave spectroscopy for diagnostics of nitrogen vacancy defects in diamond samples
}

\author{
D. Rudnicki, ${ }^{* 1}$ M. Mrózek, ${ }^{1}$,J. Młynarczyk, ${ }^{2}$ and W. Gawlik ${ }^{1}$ \\ ${ }^{1}$ Institute of Physics, Jagiellonian University, Reymonta 4, 30-059 Krakow, Poland \\ ${ }^{2}$ Department of Electronics, AGH University of Science and Technology, Krakow, Poland
}

Received October 29, 2013; accepted December 17, 2013; published December 31, 2013

\begin{abstract}
A setup is described that combines microwave spectroscopy with optical excitation and detection of luminescence of $\mathrm{NV}^{-}$colour centers in diamond crystals. The parameters of the system (design of the microwave antenna, microwave power and timing, and fast optical detection) allow studies of relaxation properties of the $\mathrm{NV}^{-}$ defects and manipulation of their spin properties.
\end{abstract}

Negatively charged nitrogen-vacancy $\left(\mathrm{NV}^{-}\right)$color centers in diamond [1] are intensively studied as promising candidates for fluorescent markers in biological systems [2], qubits that can be optically initialized and read out [3], and sensors of magnetic fields [4]. The NV center is a point defect in the diamond lattice. It consists of a pair of a substitutional nitrogen atom and a lattice vacancy next to it.

The magnetometric applications of $\mathrm{NV}^{-}$centers exploit their paramagnetic properties associated with an electronic spin $S=1$ and possibility of addressing it by a microwave (MW) field of about $2.88 \mathrm{GHz}$ [5]. One of the main tools which is used for NV research are the coherently driven electron spin oscillations (the Rabi oscillations). The first observation of Rabi oscillations in $\mathrm{NV}^{-}$color centers was reported in 2004 by F. Jelezko et al. [5]. Since then the method has been widely applied for studying the interaction of $\mathrm{NV}$ centers with external fields.

In this work, we describe our study of Rabi oscillations in a system of optically oriented $\mathrm{NV}^{-}$centers. We focus on optimization of the Rabi oscillations by a custom designed high-performance microwave antenna.

The experiment was carried out at room temperature with a type IIa bulk diamond (HPHT diamond produced by Element 6). The sample was selected with a very high nitrogen density ( 200ppm), irradiated by an electron beam with $10^{18} \mathrm{~cm}^{-2}$ dose, and annealed for 4 hours at $650^{\circ} \mathrm{C}$. Figure 1 depicts our setup. The $2 \times 2 \times .5 \mathrm{~mm}$ diamond sample is mounted close to the microstrip MW antenna (described below and presented in Fig. 3) and is continuously excited by a focused $532 \mathrm{~nm} \mathrm{CW}$ laser beam of $1-100 \mathrm{~mW}$ power. The beam is focused by a microscope $(40 \times)$ objective, or an aspheric lens with focal length of 7$9 \mathrm{~mm}$ ). This excitation polarizes the $\mathrm{NV}^{-}$ground-state spins

\footnotetext{
*E-mail: daniel.rudnicki@uj.edu.pl
}

by optical pumping and creates intense red fluorescence of the $\mathrm{NV}^{-}$centers at the $637 \mathrm{~nm}$ wavelength. The fluorescence light is transmitted by the same confocal lens system as the green exciting beam, is spectrally filtered by the dichroic mirror, and recorded by a photodiode.

The optical pumping of the $\mathrm{NV}^{-}$ground state occurs due to spin-dependent intersystem singlet-triplet crossing. This crossing effectively transfers the NV centers with the spin projection $m= \pm 1$ to the $m=0$ ground-state sublevel via the ${ }^{1}$ A state (Fig. 2a). Consequently, the ground state of $\mathrm{NV}^{-}$ becomes spin-polarized and may be interrogated by a resonant MW field.

In order to change the spin state and perform MW spectroscopy, $4 \mu$ s MW pulses, resonant with the $m= \pm 1 \leftrightarrow$ $m=0$ transition, are applied with a repetition rate of $100 \mathrm{~Hz}$. When the MW frequency matches the separation of the $m$ sublevels, an optically-detected microwave resonance (ODMR) is observed as about a $1-5 \%$ drop of the fluorescence intensity (Fig. 2b).

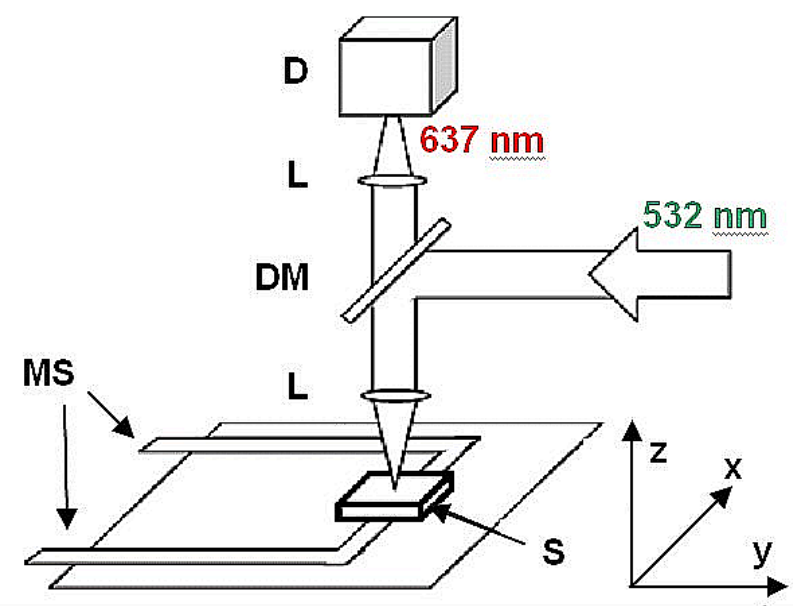

Fig. 1. The experimental setup for microwave spectroscopy of optically pumped NV diamonds. S denotes the diamond sample, MS - the microstrip antenna, $\mathrm{L}$ - the lens system, DM - the dichroic mirror, and $\mathrm{D}-$ the photodiode. Neither the electronic circuitry, nor the $532 \mathrm{~nm}$ CW laser are shown. 
(a)

(b)

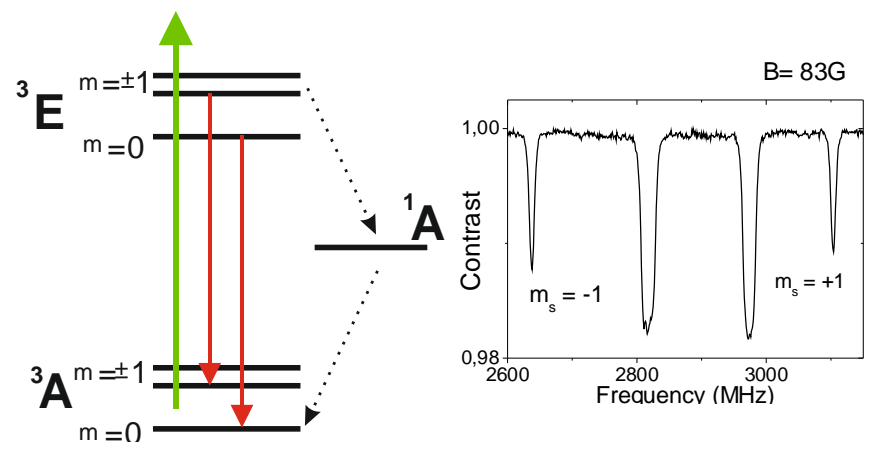

Fig. 2. a) Energy level scheme of the NV center in diamond.

b) Optically detected magnetic resonance (ODMR) in a magnetic field of $83 \mathrm{G}$.

The antenna circuit that allows generation of a strong local magnetic field for driving the MW transitions at $2.88 \mathrm{GHz}$ consists of a microstrip line of a specially designed shape and was fabricated on an insulating FR4 substrate (Fig. 3a). The bottom substrate layer is covered by the grounded plane. Two SMA connectors enable connecting an RF generator and a $50 \Omega$ load.

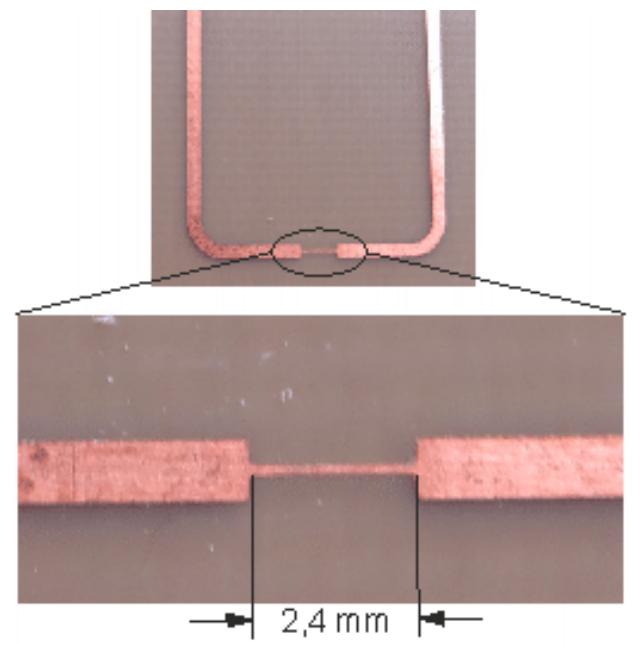

(a)
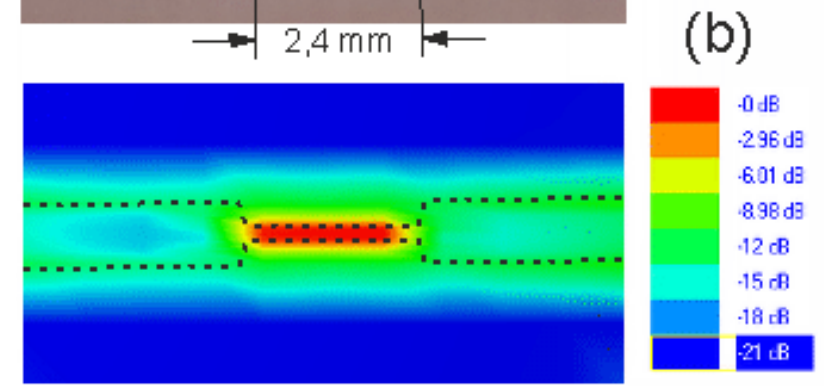

Fig. 3. (a) Photograph of the PCB microstrip line which drives the MW transition in the NV sample and its enlarged detail. (b) The simulation of the magnetic field distribution $0.1 \mathrm{~mm}$ above the top layer of the PCB (the $\mathrm{B}_{\mathrm{z}}$ component). Only the upper middle part is shown. The field strength is the highest in the vicinity of the narrow part of the microstrip line where the sample is placed.
The narrow part of the microstrip line in the upper part of the PCB produces strong $B_{y}$ and $B_{z}$ components of the magnetic field in the $x-y$ plane. Figure $3 \mathrm{~b}$ shows the simulation of the magnetic field distribution above the upper part of the PCB. The sample is placed on top of the narrow part of the microstrip line, in the area where the magnetic field strength is the highest. The described antenna improved the intensity of the MW field by more than one order of magnitude with respect to the standard, non-optimized single wire arrangements.

For good separation of the $m=-1$ and $m=+1$ states of the $\mathrm{NV}$ ground state, a static magnetic field of $83 \mathrm{G}$ was applied along the [111] crystallographic orientation of the diamond sample, resulting in the ODMR spectrum shown in Fig. 2b. With that magnetic field and sufficiently strong MW pulses, the Rabi oscillations of the fluorescence intensity were detected with a fast avalanche photodiode. Figure 4 presents an example of Rabi oscillations and a mathematical fit of the fluorescence intensity to the decaying sinus function.

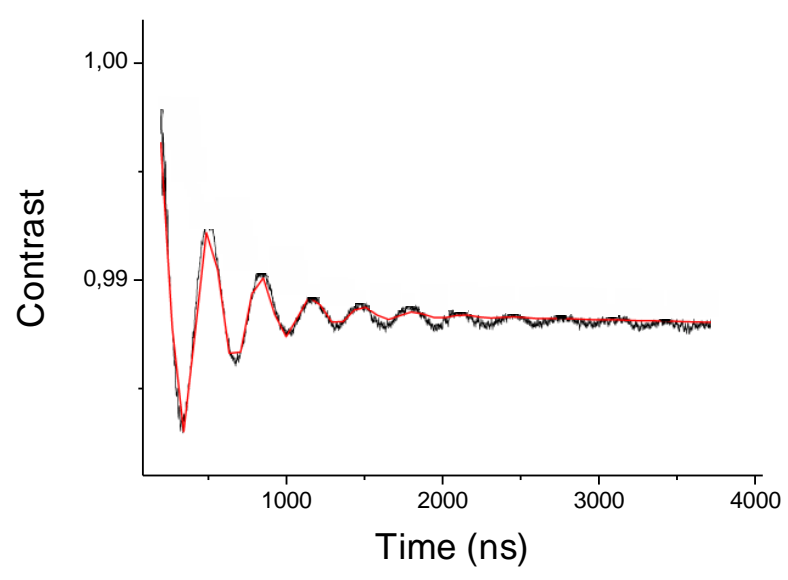

Fig.4. Rabi oscillation in one of the studied samples (E1). The black curve is the experimental data while the red one is a fit to the $C(t)$ function (described in the text) for $T=830 \mathrm{~ns} \pm 10 \mathrm{~ns}$ and $w=360 \mathrm{~ns} \pm 19 \mathrm{~ns}$.

The Rabi oscillations were recorded for different microwave power levels. The dependence of the oscillations frequency on the MW power appears to be linear (Fig. 5 inset), in agreement with the theory of coherent oscillations in a two level system. The range of the frequencies of the observed Rabi oscillations was between 0.58 and $3.1 \mathrm{MHz}$. The decay time of the oscillations is about two times shorter than that observed in single NV centers [5]. We attribute this difference to inhomogeneities in a bulk HPHD diamond sample. The data were fitted to the function $C(t)$ :

$$
C=C_{0}-b t+\sin \left(2 \pi\left(t-t_{c}\right) / w\right) \exp (-t / T),
$$

where: 
$C$ is the ODMR contrast, i.e. the normalized fluorescence level with the MWs on and off, $C_{0}-b t$ is the mean fluorescence level (slowly time-dependent, $b \sim 10^{-7}$ ), $t_{\mathrm{c}}$ is the initial oscillation phase, $w$ the oscillation period, and $T$ the decay time.

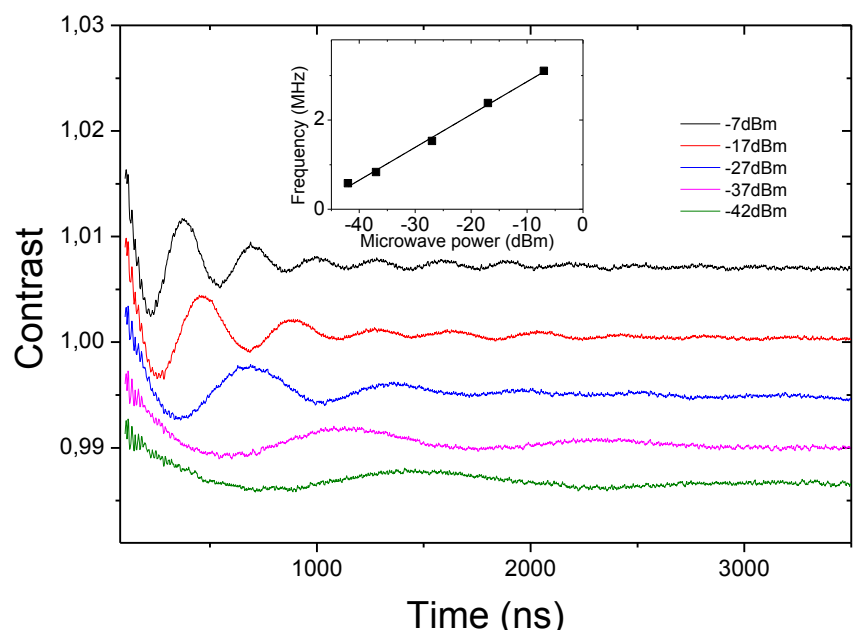

Fig. 5. Rabi oscillations recorded for various MW powers in sample E1 with a magnetic field of $83 \mathrm{G}$ along the [111] direction.

We have also studied the dependence of Rabi oscillations on the pumping laser power. We found that the Rabi frequency decreases with the laser power (Fig.6). This observation remains yet to be explained.

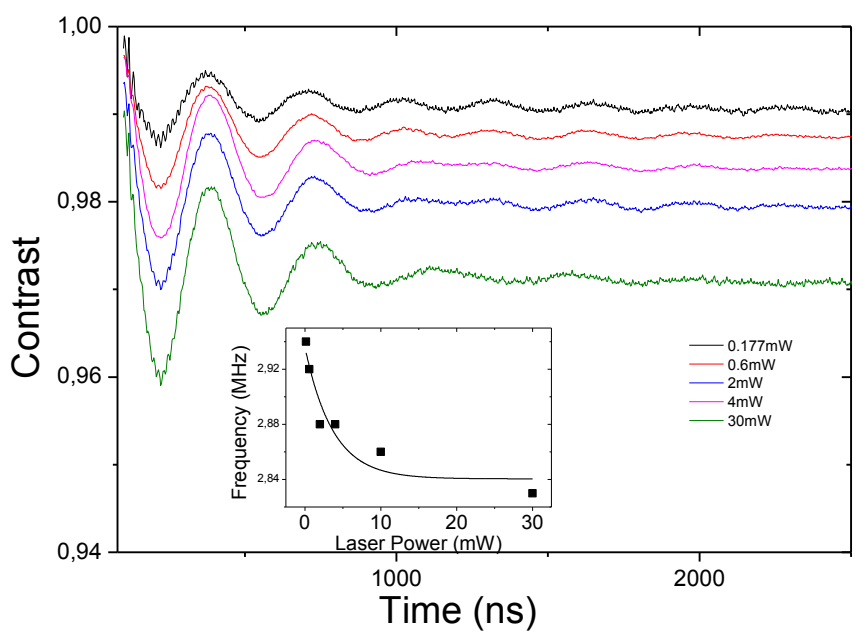

Fig. 6. Rabi oscillations recorded for various powers of the $532 \mathrm{~nm}$ laser (in sample E1 with magnetic field of $83 \mathrm{G}$ along direction [111]).
In conclusion, we have presented the simple setup for studies of $\mathrm{NV}^{-}$colour centers in diamond crystals, which combines microwave spectroscopy with optical excitation and detection of luminescence. The custom design of the microwave antenna allowed creating MW fields more than one order of magnitude stronger than in standard designs. The parameters of the system (MW power and timing and fast optical detection) allow applications of the setup for studies of relaxation properties of the $\mathrm{NV}^{-}$defects. In particular, accurate determination of the Rabi oscillations yield values of the local intensities of the local MW fields which are indispensable for precision measurements and control of the $\mathrm{NV}^{-}$spins. Moreover, we have demonstrated that relaxation times in a bulk $\mathrm{NV}^{-}$samples are about two times shorter than those in individual colour centers.

The project has been partially supported by the FNP TEAM program, MNSW grant 7150/E-338/M/2013, and the Science for Peace NATO Program.

\section{References}

[1] C. Bradac, T. Gaebel, J.R. Rabeau, Nitrogen-Vacancy Color Centers in Diamond: Properties, Synthesis, and Applications, in Optical Engineering of Diamond (eds R.P. Mildren and J.R. Rabeau, WileyVCH Verlag GmbH \& Co. KGaA, Weinheim, Germany 2013), doi: 10.1002/9783527648603.ch5

[2] C.-C. Fu, H.-Y. Lee, K. Chen, T.-S. Lim, H.-Y. Wu, P.-K. Lin, P.K. Wei, P.-H. Tsao, H.-C. Chang, W. Fann, Proc. Natl. Acad. Sci. U.S.A. 104, 727 (2007)

[3] P. Neumann, R. Kolesov, B. Naydenov, J. Beck, F. Rempp, M. Steiner, V. Jacques, G. Balasubramanian, M.L. Markham, D.J. Twitchen, S. Pezzagna, J. Meijer, J. Twamley, F. Jelezko, J. Wrachtrup, Nature Phys. 6, 249 (2010).

[4] J.M. Taylor, P. Cappellaro, L. Childress, L. Jiang, D. Budker, P.R. Hemmer, A. Yacoby, R. Walsworth, M.D. Lukin, Nature Phys. 4 810 (2008).

[5] N.R.S. Reddy, N.B. Manson, E.R. Krausz, J. Luminescence 38, 46 (1987).

[6] F. Jelezko, T. Gaebel, I. Popa, A. Gruber, J. Wrachtrup, Phys. Rev. Lett. 92, 076401 (2004). 